\title{
Enseñanza y apropiación didáctica del yaruro, lengua ancestral de los pumé, un pueblo indígena originario de Venezuela
}

\author{
Yuraima L. RodRÍGUEz BLANCO \\ Universidad Alfonso X el Sabio \\ Agustín de la Herrán Gascón \\ Universidad Autónoma de Madrid
}

Recibido: 23 agosto 2013 / Aceptado: 10 agosto 2014

ISSN:1697-7467

\begin{abstract}
RESUMEN: Este estudio se refiere a la enseñanza del yaruro, lengua originaria de los pumé, un pueblo indígena venezolano. Además, aborda cuestiones relacionadas: contextuales, lingüísticas, metodológicas, interculturales, de formación del profesorado, etc. Pretende profundizar en la comprensión de factores que intervienen en la apropiación didáctica de esta lengua, investigar sus relaciones y diseñar y evaluar una guía didáctica que mejore su enseñanza. La investigación es empírica, de corte etnográfico-fenomenológico y se ha desarrollado en todas las escuelas de enclaves pumé cuya lengua materna es el yaruro. Los resultados señalan que una educación dinámica fundada facilita la apropiación de la lengua originaria. Palabras Claves: Educación intercultural bilingüe, lengua indígena originaria, formación del profesorado, indígenas pumé, apropiación didáctica de la lengua indígena originaria.
\end{abstract}

Teaching and educational appropriation of Yaruro, the ancestral language of the Pumé, an indigenous people native to Venezuela

\begin{abstract}
This study concerns Yaruro teaching, Pumé native language of a Venezuela indigenous people. It addresses issues: contextual, linguistic, methodological, cultural, teacher training, etc. it aims to deepen the understanding of factors involved in the appropriation of the language teaching, investigate their relationships and design and evaluate an educational guide to improve their teaching. The research is empirical, phenomenological ethnographic cutting and developed in all schools Pumé enclaves whose mother language is Yaruro. The results show that a dynamic education founded facilitates ownership of the native language.
\end{abstract}

Keywords: Intercultural bilingual education, native indian language, teacher training, indigenous pumé, appropriation language teaching native indigenous.

\section{INTRODUCCIÓN}

La investigación se desarrolló durante cuatro años (de junio de 2009 a junio 2013) en las escuelas pumé. Estas escuelas pertenecen a la Red de Escuelas Indígenas Bolivarianas Guaicaipuro", que se localiza en el municipio "Pedro Camejo" del Estado Apure (Venezuela). Para llegar a estas escuelas hay que viajar por carretera, río y a pie. La dificultad de acceso 
es tal que favorece su aislamiento. No es un destino turístico. Cuando alguna vez llegan turistas, hablan con ellos, les graban y se van sin ayudarles. Sus problemas son graves. El mayor tiene que ver con su existencia allí, con la preservación de su cultura y del yaruro, su lengua. La cultura pumé incluye su identidad, sus relaciones con la tierra, con la selva y con su gente en el mundo de hoy para el mañana. Su lengua es la clave para comunicarse y no desaparecer. Saben bien que, cuantas más personas hablen y escriban yaruro, mayores serán las posibilidades de permanecer y auto organizarse ante las presiones e influencias de los entornos culturales dominantes. Por ello, esta investigación es a la vez minoritaria y vital.

Para los pumé la educación intercultural bilingüe de sus niños está vinculada a su supervivencia. Por eso es imprescindible una acción pedagógica orientada al fortalecimiento de su enseñanza para la apropiación didáctica del yaruro. La inacción puede llevar al deterioro y a su extinción, por una suerte de ósmosis desigual entre la cultura pumé y la cultura mayoritaria e indiferente. Se hace imprescindible la aplicación de políticas y planes educativos asociados a la formación de su profesorado bilingüe, la metodología, los recursos didácticos y la participación de las comunidades. Pero estas políticas aplicables a la problemática pumé no existen. En esta situación, los profesores bilingües podrían ser decisivos: conocen la lengua originaria y la lengua de la cultura dominante (castellano), conocen la cultura de sus alumnos. De ellos podría depender la calidad de la formación que, inicialmente y en lo que a su lengua materna se refiere, tenderá a ser espontánea: "El niño y la niña se van apropiando de la lengua de una forma natural, mediante experiencias de lenguaje que se presentan en las situaciones sociales cotidianas y de juego que tienen sentido para él y para ella" (Ruiz, 1996: 48). Este proceso se corresponde con su uso desde los primeros años de vida en el hogar, la escuela y la comunidad. En la escuela el niño continuará desarrollando competencias en su aprendizaje asociadas a las normas de escritura, lectura y entonación de su lengua. Por ello la enseñanza debe enraizarse en su entorno para obtener resultados significativos y relevantes:

Aprender una lengua es un proceso dinámico y creativo. De ahí que, como profesores preocupados por mejorar nuestra práctica docente, hemos de procurar crear situaciones auténticas dentro del aula, de modo que se refleje el uso de la lengua que encontramos en contextos reales de comunicación (Martínez, 2007:41).

Todo ello sin menospreciar la lengua del grupo cultural dominante (Haugen, 1972; Calvet, 1999; Bondarenko, 2010), ya que, desde una perspectiva formativa y práctica, será su equilibrio con la lengua materna y el reconocimiento de la importancia de ambas los que facilitarán que el niño adquiera destrezas que le permitan comunicarse en cualquier entorno.

Para la realización de este trabajo se han considerado antecedentes relevantes nacionales e internacionales. La mayoría de investigaciones realizadas en España sobre interculturalidad no tienen relación con el entorno indígena, debido a la carencia de ese colectivo en el país. Pero hay trabajos españoles que desarrollan el trabajo de campo en países latinoamericanos que sí cuentan con ellos. Los que no optan por esta vía basan sus estudios en el análisis de las comunidades inmigrantes y extranjeras residentes en España, tomando aspectos como su interrelación, integración en la sociedad, su estilo de vida, valores, costumbres y tradiciones, entre otros. En cuanto a los estudios internacionales, si bien se tienen en cuenta las aportaciones relativas a los fundamentos de la educación intercultural (Abdallah-Pretceille, 
1986, 1996; Camilleri y Cohen-Emerique, 1989; Demorgon, y Lipianski, 1999), los que se han considerado más pertinentes son los relativos a la Educación Intercultural Bilingüe en contextos indígenas. Los estudios consultados se sintetizan en el gráfico $\mathrm{N}^{\circ} 1$.

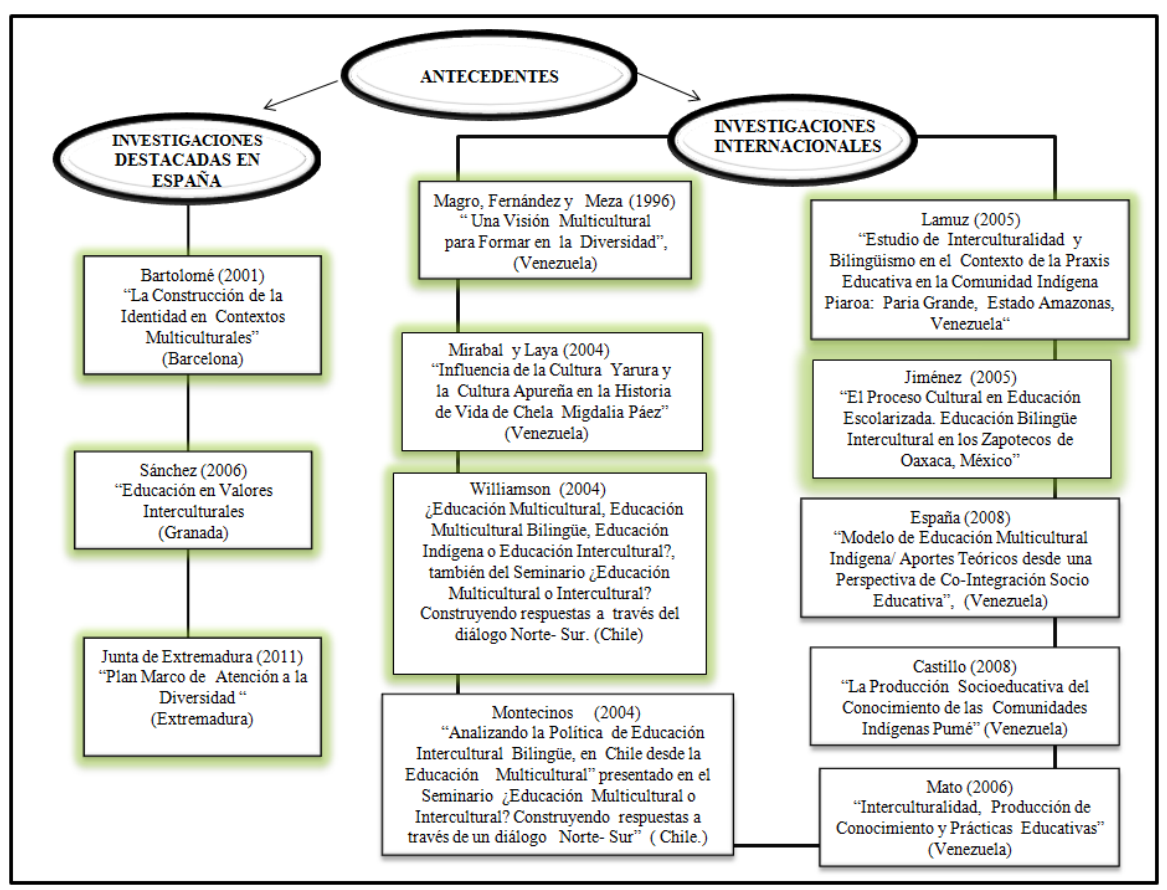

Gráfico $N^{o}$ 1: Antecedentes de la Investigación

\section{ObJetivos}

La investigación intenta satisfacer dos objetivos generales:

1) Profundizar en la comprensión de la problemática asociada a la enseñanza y la apropiación didáctica del yaruro en el marco de la educación intercultural bilingüe en la escuela primaria pumé.

2) Elaborar, aplicar y evaluar una guía didáctica que facilite su enseñanza en este contexto.

Estos objetivos generales se concretan en los siguientes seis objetivos específicos:

1) Definir referentes teóricos válidos para fundamentar la enseñanza y la apropiación didáctica de las lenguas indígenas originarias en el marco de la educación intercultural bilingüe aplicables al contexto de investigación. 
2) Identificar y comprender en profundidad la problemática asociada al proceso de enseñanza y apropiación didáctica del yaruro en las escuelas pumé de enseñanza primaria.

3) Interpretar críticamente el desarrollo del modelo de educación intercultural bilingüe aplicada por los docentes de educación primaria en las escuelas pumé.

4) Descubrir y sistematizar categorías emergentes de la investigación que evalúen y resignifiquen la enseñanza y apropiación didáctica del yaruro en su contexto.

5) Deducir implicaciones pedagógicas para la formación de los profesores de educación intercultural bilingüe para la didáctica del yaruro en la etapa primaria.

6) Elaborar, aplicar y evaluar una guía didáctica que pueda facilitar la enseñanza y la apropiación de las lenguas indígenas originarias, con proyección al ámbito indígena latinoamericano.

\section{Marco contextual y participantes}

La Red de Escuelas Indígenas Bolivarianas "Guaicaipuro" se ubica en el municipio "Pedro Camejo", del Estado Apure (Venezuela), en el Suroeste del país. Los participantes del estudio fueron los docentes de educación primaria que trabajan en las nueve escuelas de la Red: "La Macanilla", "Piedra Azul", "Agua Linda", "Médano Alto", "Playa Grande", "Copa de Oro", "Santa Josefina", "Caño La Guardia" y "San Isidro". En el transcurso de la investigación se detectó que las dos últimas escuelas "Caño la Guardia" y "San Isidro" tenían como lengua indígena predominante el jivi, porque entre los años 2009 y 2013 no contaban con alumnos de habla yaruro. Esta fue la razón por la que el estudio se circunscribió sólo a siete de las nueve escuelas indígenas bolivarianas de la Red "Guaicaipuro" y a sus quince docentes en activo. En su mayoría estos docentes no dominaban la lengua yaruro, por lo que empleaban el castellano para enseñarla. Mostraban, además, una importante falta de formación pedagógica en Educación Intercultural Bilingüe, a pesar de poseer estudios universitarios en el área de la Didáctica.

Las escuelas se ubican en comunidades indígenas y en zonas rurales de difícil acceso, distantes entre sí. Este hecho actúa a favor de su aislamiento y continuidad. Su hábitat son los márgenes de ríos, caños y lagunas. Al existir una amplia distancia entre cada comunidad, para desarrollar la investigación, se consideró conveniente pernoctar varias semanas en cada visita realizada, adaptándose al medio y a sus complejas, variadas y extremas condiciones climáticas: fuertes lluvias, que provocaban inundaciones, y amplias temporadas de sequía, que impedían el desplazamiento por los ríos. Estas circunstancias condicionaron los viajes y el desarrollo del estudio.

\section{Fundamentación teórica}

\subsection{Retos sociales y educación intercultural}

La educación es un pilar fundamental de la evolución social. Como proceso global pretende la formación del individuo y la mejora social, orientados a un mejor futuro. Su complejidad responde tanto a retos perennes, radicales, básicos para la formación, no con- 
textuados y que socialmente no se demandan (Herrán, 2011), como a desafíos culturales y multiculturales fuertemente enraizados, orientados tanto a su preservación como a su renovación. Las necesidades del estudio se inscriben en este segundo grupo de retos sociales, y se justifican en el hecho multicultural. Al respecto, Leal (2010:23) sostiene que:

El hecho multicultural es una característica cada vez más presente en las sociedades actuales; la educación no puede por tanto, obviar esta realidad y debe plantearse la necesidad de cambios así como el compromiso de planificar metodologías más sensibles que den respuesta a las crecientes demandas de la escuela multicultural.

El hecho multicultural puede percibirse con una perspectiva intercultural. Con este enfoque, la práctica educativa se concreta en actuaciones motivadas por un profundo respeto a la diferencia y por un compromiso de encuentro entre la identidad y la diversidad cultural. La metodología general de ese encuentro es el conocimiento mediante el diálogo intercultural, que puede enriquecer a sus participantes, personal y socialmente. Este camino educativo se orienta hacia una sociedad más justa, cívica y democrática, basada en el respeto y la educación de sus participantes. Con esta perspectiva, la educación intercultural puede conceptuarse como:

Un proceso de formación que desarrolla en las personas las capacidades para desenvolverse con éxito en sociedades multiculturales, adquirir habilidades y conocimientos a lo largo de su vida en cualquier ámbito cultural, mantener una actitud de aceptación y valoración de la diversidad (García, 2009:146).

\subsection{Educación intercultural bilingüe y enseñanza}

La educación intercultural no es una nueva ideología, sino una respuesta democrática y pedagógica a las necesidades de las plurales sociedades actuales. Pero además puede ser una alternativa posible para afrontar los desafíos de las sociedades multiculturales. A veces, ayuda a superar fracasos en los actuales sistemas educativos. Como enfoque didáctico, busca propiciar el enriquecimiento cultural de los ciudadanos, partiendo del reconocimiento y respeto a su diversidad y a lo que les es propio a través del intercambio, el diálogo y el desarrollo de una sociedad democrática, tolerante y solidaria (García, 2009: 67, adaptado).

En los contextos minoritarios como el pumé, con lengua indígena ancestral, la educación intercultural adquiere un marcado acento bilingüe. Su éxito es imprescindible para su preservación. El apoyo de la comunidad, la formación pedagógica del docente, el dominio de la lengua originaria y de su didáctica y el conocimiento del entorno indígena son factores de su desarrollo. Su presencia puede otorgar al docente bilingüe las herramientas necesarias para desarrollar, a través de su lengua, una praxis didáctica contextuada, amena, con contenidos adecuados, y capaz de cubrir las necesidades de los niños indígenas y de su cultura. Coincidimos con Ramos (2010: 56) cuando expone:

Para definir lo que se debe enseñar, en primer lugar, es necesario conocer el contexto legal y social del lugar en el que se pretende desarrollar la docencia [...] Los conocimientos sobre el contexto social son importantes para adaptar esos contenidos a la realidad en la que viven los alumnos. [...] La adaptación de los contenidos al medio en el que vive el alumnado produce mejores resultados en el aprendizaje. 


\section{MÉtodo de investigación}

\subsection{Enfoque y diseño de investigación}

Para cumplir los objetivos se optó por una metodología cualitativa, conceptuada por Hernández Sampieri (2006: 30) como:

Un conjunto de prácticas interpretativas que hacen al mundo visible, lo transforman y convierten en una serie de representaciones en forma de observaciones, anotaciones, grabaciones y documentos... Es interpretativo porque intenta encontrar más sentido a los fenómenos en términos de los significados que las personas les otorguen.

Su orientación fue fenomenológica-hermenéutica. Para la fenomenología, el mundo que conocemos es el mundo tal como se nos ofrece a la conciencia que lo desarrolla (Husserl, 1990). Por eso la investigación se basó en el estudio de las experiencias vividas desde la percepción de los participantes:

La fenomenología considera que los seres humanos están vinculados con su mundo y pone énfasis en su experiencia vivida, la que aparece en el contexto de las relaciones con objetos, personas, sucesos y pretende entender los fenómenos sociales desde la propia perspectiva del actor (Taylor y Bogdan, 2000:35).

El empleo de una suficiente variedad de técnicas e instrumentos de recogida de datos fue una decisión acorde con el enfoque de investigación que permitió satisfacer los objetivos planteados. Para procesar y analizar la información se recurrió a la teoría fundamentada (grounded theory) (Glaser, 1978; Corbin, y Strauss, 1990), basada en la comparación constante. Globalmente, en la medida en que el diseño incluye la elaboración de una guía didáctica que se aplica y evalúa con posibilidades de ciclos posteriores de evaluación-planificación-acción, cabe conceptuarse como investigación-acción participativa (Villasante, 2006).

El estudio se realizó en un tiempo aproximado de cuatro años, de junio de 2009 a junio de 2013. Durante el primer año se procedió a una investigación documental. Los dos años consecutivos se aplicaron las técnicas de recolección de datos: observación participante y entrevista en profundidad. Se realizaron un total de seis visitas a cada una de las siete escuelas. El año siguiente se realizó el grupo de discusión y el taller formativo de los docentes, así como el diseño, aplicación y evaluación de la guía didáctica correspondiente al último objetivo de la investigación.

\subsection{Técnicas e instrumentos de producción y recogida de datos}

Se utilizaron varias técnicas complementarias: observación participante, entrevista en profundidad y grupo de discusión. La información se registró en los siguientes instrumentos: diario de investigación, notas de campo, lista de comprobación o de cotejo y grabaciones. La diversidad de técnicas e instrumentos de recolección de datos fue una respuesta a la complejidad del escenario de investigación y a la búsqueda de la mayor validez posible de cara a su análisis interpretativo. 
Se recurrió a la observación participante para profundizar en la comprensión del contexto y el problema estudiado:

La meta para el diseño de la investigación usando la observación participante como un método es desarrollar una comprensión holística de los fenómenos en estudio que sea tan objetiva y precisa como sea posible, teniendo en cuenta las limitaciones del método (Marshall y Rossman, 1995:92).

Su desarrollo a través de observaciones continuas y abundantes fue más fácil por la condición de miembro del pueblo pumé de la investigadora. Para aumentar su validez se realizó un alto número de observaciones en lapsos dilatados. De acuerdo con Marshall y Rossman (1995), su validez se puede incrementar al combinarse con otras técnicas, tales como las entrevistas, el análisis de documentos, las encuestas, los cuestionarios u otros instrumentos. A la vista de ello, nuestra opción fue combinarla con la entrevista en profundidad y el grupo de discusión.

La entrevista en profundidad estuvo determinada por el enfoque fenomenológico de la investigación. Se desarrollaron como "reiterados encuentros, cara a cara, entre el investigador y los informantes, encuentros éstos dirigidos hacia la comprensión de las perspectivas o situaciones, tal como las expresan con sus propias palabras" (Taylor y Bogdan, 2000:67). Para su desarrollo se perfiló una somera guía de entrevista previamente validada que sirvió como recordatorio, que fue útil en la evaluación interpretativa para sistematizar lo que se abarcó con los participantes:

Se trata de una lista de áreas generales que deben cubrirse con cada informante, no es un protocolo estructurado, en el que el investigador decide cómo enunciar las preguntas y cuándo formularlas, sirve solamente para recordar que se deben hacer preguntas sobre ciertos temas (Patton, 1990: 54).

El grupo de discusión se conceptuó como "una forma de acercamiento inicial a los datos que emergen en el proceso de reflexión de los participantes y que permiten obtener un marco general para la comprensión de los mismos" (Mena, 2009: 59). Se desarrolló también sobre una guía didáctica, comprendida como: "secuencia de las temáticas o preguntas que serán presentadas a los participantes durante las sesiones de discusión" (Krueger, 1988: 56). La guía se validó previamente con expertos y una prueba piloto. Incluía la relación de participantes y los objetivos que se deseaban cubrir. Sus desarrollos didácticos abarcaron seis temáticas o preguntas abiertas y oscilaron entre una y dos horas. Para registrar los datos se utilizó el diario de campo, comprendido como "eje que articula las dimensiones empírica y teórica de la investigación, la cual se construye como instrumento, presentando utilidades de alcance general" (García, 2004:244).

Se empleó para registrar hechos como percepciones, intuiciones y sentimientos del investigador, problemas, dificultades y puntos fuertes del estudio, estrategias fallidas y efectivas, cambios en los guiones, resolución de conflictos, etc. ya ocurridos que son susceptibles de interpretación. También se recurrió a las notas de campo, comprendidas por Hammersley y Atkinson (1994:162) como: 
Descripciones de los procesos sociales en los contextos donde suceden y anotaciones reflexivas sobre la aplicación de estrategias y técnicas de investigación, cuya finalidad es captar procesos sociales en su integridad, resaltando sus diferentes características y propiedades en función de cierto sentido común sobre lo que es relevante para los problemas planteados en la investigación.

Permitieron registrar características y peculiaridades de tales procesos, reconstruir la visión del investigador, sus relaciones con los actores y los escenarios y dar cuenta de la memoria metodológica del proceso investigativo. Además, se empleó la lista de comprobación o de cotejo, "un instrumento de la técnica de observación caracterizado por presentar una serie de aspectos a corroborar y facilitar el detalle sobre algunas variables o categorías" (Casal, 2006: 65). Con ella se registró la frecuencia de los eventos observados. El cuarto soporte de registro fueron las grabaciones (fotografía, vídeo y audio), previo consentimiento informado de los participantes y de las familias de los niños. La fotografía se utilizó para retratar la realidad de manera objetiva. El vídeo se empleó por tratarse de un soporte "fácil de codificar a efecto de un análisis posterior" (Busot, 1991:124). Aun cuando Taylor y Bogdan (2000:130) no aconsejan el uso de la grabación en la observación participante, sostienen un punto de vista distinto cuando se trata de la entrevista. Un grabador de audio "permite al entrevistador captar mucho más que si se valiera únicamente de su memoria, ya que los datos del entrevistado son casi exclusivamente palabras".

\subsection{Técnicas de análisis e interpretación de datos}

Las técnicas de análisis empleadas fueron coherentes con el enfoque y el diseño de la investigación. Abarcaron la triangulación metodológica, la categorización, la teorización y el análisis de la teoría fundamentada.

La triangulación metodológica es la confirmación de datos obtenidos desde múltiples técnicas sobre un mismo objeto de estudio. Para Leal (2005:116) es: "la determinación de ciertas intersecciones o coincidencias a partir de diferentes apreciaciones y fuentes informativas o varios puntos de vista del mismo fenómeno".

La categorización es la tarea de análisis de datos cualitativos más representativa y habitual, consistente en su reducción, sistematización e interpretación rigurosa. Martínez (1991: 63) la considera "un proceso descriptivo que se realiza una vez aplicados los instrumentos, para elaborar definiciones de lo expresado por los informantes y luego interpretar los términos, que son agrupados en subcategorías".

La teorización envuelve la construcción de relaciones procesos, dimensiones y emergencias para generar teorías sobre el modelo subyacente en las prácticas educativas. Martínez (2004: 73) sostiene que la teorización en un estudio de corte cualitativo viene dada por la construcción mental simbólica, verbal o icónica de naturaleza conjetural o hipotética, donde se induce a repensar de un modo nuevo, a completar, integrar, unificar, sistematizar o interpretar un cuerpo de conocimientos que hasta el momento se consideraban incompletos, imprecisos, inconexos o intuitivos.

La teoría fundamentada o grounded theory procesa la información desde un "método comparativo constante" (Dey, 1999). Su objeto es formar categorías, valorar y verificar su pertinencia, asignarlas al texto y contrastar incidentes mediante el rastreo de evidencias 
(Bryant y Charmaz, 2007). Pretende discernir similitudes conceptuales, refinar el poder discriminatorio de las categorías y descubrir patrones. Esta metodología permitió codificar y analizar los datos para desarrollar conceptos.

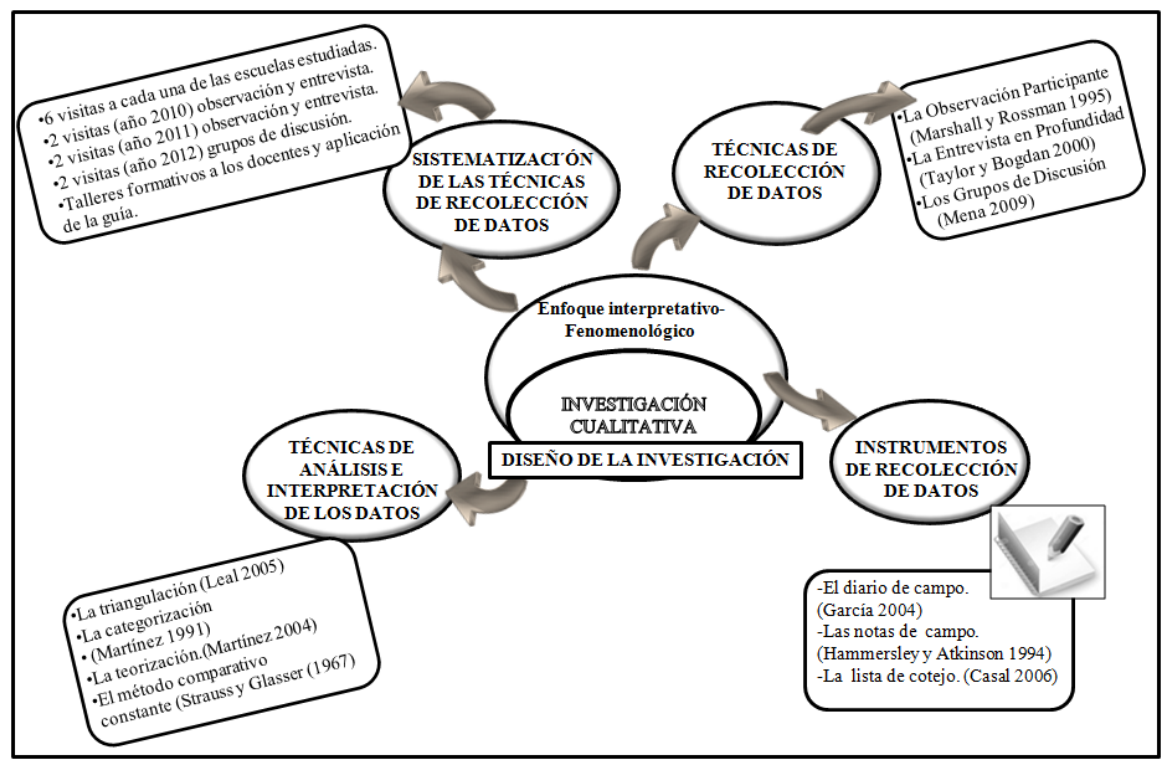

Gráfico $N^{o}$ 2: Método de investigación

\section{Análisis de los resultados}

\subsection{Resultados contextuales}

Los datos recogidos definieron problemas contextuales interrelacionados de carácter estable, conceptuables como condicionantes de la enseñanza y apropiación didáctica de la lengua yaruro:

a) Currículo oficial inadecuado para la realidad indígena.

b) Inexistencia de un modelo de educación intercultural bilingüe dirigido al entorno indígena.

c) Falta de formación didáctica del docente en esta área.

d) Falta de dominio de la lengua originaria por parte de los profesores que enseñan a los pumé.

e) Pobreza metodológica en la comunicación didáctica. Esto es, falta de aplicación de técnicas de enseñanza motivadora y formativa y escasez de recursos didácticos.

f) Escasa integración entre la escuela y la comunidad. 


\subsection{Resultados desde las categorías de análisis}

Los resultados contextuales anteriores se relacionan con los obtenidos desde las categorías emergentes del estudio: dos de carácter curricular, tres culturales-contextuales y cuatro categorías relativas a la enseñanza:

\subsubsection{Categorias curriculares}

- Categoría 'currículum': El docente bilingüe de yaruro se apoya en el Currículum Básico Nacional (C.B.N.), normativa legal vigente de carácter didáctico. Lo comprende y utiliza de dos modos: $1^{\circ}$ ) Como conjunto de asignaturas que organizan intenciones de enseñanza, entre las que destacan los contenidos. Aunque se ciñen a ellos, es consciente de que algunos no son pertinentes con el contexto indígena. Este hecho le mueve a hacer adecuaciones curriculares poco pertinentes. La aplicación curricular al aula tiende a la literalidad. $2^{\circ}$ ) Como guía de tareas y actividades. El profesor bilingüe de las escuelas estudiadas emplea el C.B.N. como guía para desarrollar actividades escolares desde una clara acriticidad, pese a reconocer, según sus valoraciones, que aquéllas no son congruentes con la realidad indígena. Esto impide una praxis contextualizada. Con este referente así empleado y con la carencia de libros de texto de alumnos, los docentes bilingües planifican las clases de yaruro sin guías docentes adecuadas y por sentido común, con apoyo de unas cartillas elaboradas por ellos.

- Categoría 'lengua indígena yaruro': Para los niños de las escuelas de la "Red Guaicaipuro", el yaruro es el medio de relación con los suyos, además de condensar su identidad social y cultural. Es un recurso instrumental con el que elaboran el sentido personal y social de su realidad, el conjunto organizado de signos que caracteriza sus emociones propias y el reflejo de sus pensamientos. El yaruro se afianza en el convivir, el conversar y en las interacciones con su entorno. Para la escuela, el yaruro es a la vez contenido de enseñanza y medio instrumental de aprendizaje. Pero en ella la significación vital del yaruro queda interrumpida. En efecto, no se percibe continuidad en el empleo de su lengua, ya que los profesores no indígenas la desconocen y algunos profesores indígenas carecen de competencias en lectura y escritura yaruras. Esta circunstancia formativa y didáctica contribuye, paradójicamente, al desplazamiento de la lengua originaria por la dominante.

\subsubsection{Categorías culturales-contextuales}

- Categoría 'integración escuela-comunidad': No hay una comunicación suficiente ni integración entre la escuela (profesores) y la comunidad (familias, ancianos, aldea), porque no se verifica: "un intercambio activo entre la institución educativa y su contexto" (Gómez, 2010: 42). Esta circunstancia favorece la continuidad del sistema y su contexto en su mismo estado. En efecto, esta falta de interrelación impide percibir y compartir problemas permanentes o circunstanciales para los que se requiere la colaboración de todos. También dificulta el encauzamiento de procesos de cambio en beneficio de los educandos y de toda la comunidad. 
- Categoría 'identidad cultural, vergüenza y orgullo étnicos': No se percibió vergüenza étnica por parte de los profesores bilingües, las familias o los niños indígenas. Al contrario, los niños indígenas viven su cultura plenamente y demostraron orgullo étnico por ser pumés. Les gusta lo que les caracteriza: hablar en su idioma, realizar artesanías, trabajar la tierra, dedicarse a los medios ancestrales de sustento, etc. Conservan su estilo de vida ancestral y el contacto con la naturaleza, reflejando el sentido de pertenencia con su pueblo. En palabras de Stuart (1996: 21), en tanto que grupo étnico específico, se percibe un intenso "conocimiento de su identidad".

- Categoría 'apropiación didáctica de la lengua indígena originaria (yaruro)': La lengua indígena originaria tiene una importante influencia en el aprendizaje de los niños indígenas. Su no utilización adecuada les aleja de su mejor uso y del resto de los aprendizajes. Si fuese bien enseñada en la escuela, potenciaría su desarrollo en los contextos educativo, familiar y social, por educarle en la expresión de su lengua materna. Los profesores bilingües no reconocen con claridad su falta de formación en el yaruro y su didáctica, pero expresan que no cuentan con conocimientos metodológicos para desarrollar este proceso.

\subsubsection{Categorías de enseñanza}

- Categoría 'formación-perfil del docente': Los docentes bilingües participantes tienen una formación universitaria no enfocada a la enseñanza del yaruro en su contexto. Su perfil no es adecuado para el desarrollo de una educación intercultural bilingüe en su entorno indígena. No dominan la lengua objeto de enseñanza, ni conocen la cultura y los valores ancestrales pumé, con los que no se identifican. Además, carecen de conocimientos metodológicos o didácticos para enseñar su lengua en su cultura. Están abiertos o sensibilizados para una formación actualizada, pero ignoran cómo conseguirla. Esta posibilidad no se oferta desde la Administración estatal. Carecen del hábito de dialogar, planificar, intercambiar experiencias o evaluar en equipo, lo que se agrava por las distancias entre las escuelas de la Red "Guaicaipuro".

- Categoría 'técnicas de enseñanza': Comprendidas bien como "concreciones metodológicas" (Herrán y Paredes, 2009), bien como "actividades creativas, variadas y diseñadas con el propósito de facilitar el desarrollo significativo de las habilidades comunicativas de los alumnos" (Wilson, 2005: 87) no eran conocidas para los docentes estudiados. No obstante, algunas de sus prácticas se aproximaban a ellas: traducciones simples, reproducciones de palabras en lengua nativa, etc. Los profesores bilingües reconocían que el efecto de sus modos de enseñar era escasamente motivador en sus alumnos: no captaban su atención, no favorecían su interés ni su concentración. Conducían a un aprendizaje repetitivo y memorístico poco significativo, que era la norma y la tradición escolar.

- Categoría 'recursos didácticos': Los recursos didácticos “propician la creación de situaciones de construcción del conocimiento interesantes y entretenidas, contribuyendo a maximizar la motivación en los estudiantes" (Martínez, 2010: 76). Los docentes bilingües manifiestan su interés por mejorar su enseñanza y la apropiación didáctica del yaruro, a través de la elaboración de recursos como la cartilla. Además 
se apoyan en el pizarrón. Ambos recursos condicionan la comunicación didáctica y dificultan el desarrollo de una praxis amena a través de otros recursos posibles más variados y atractivos para los estudiantes.

- Categoría 'praxis pedagógica bilingüe': Los maestros bilingües enfocan su praxis en la enseñanza de la gramática yarura. En su didáctica no parece influir que se enseñe a niños indígenas o no indígenas. Su enseñanza se caracterizó por tres ausencias didácticas: no hubo planificación de las clases, no se recurrió a una metodología didáctica elaborada y no se desarrolló una evaluación didáctica sistemática. Desde un punto de vista personal, los docentes de aula no se integran en los procesos formativos con sus alumnos. Todo ello es causa de un clima didáctico pobre, de aprendizajes aislados y poco coherentes entre sí y de una falta de orientación didáctica global.

\subsection{La guía didáctica y sus resultados}

La guía didáctica tuvo como destinatarios a los docentes bilingües de primaria de las siete escuelas pumés estudiadas. Las fuentes para su diseño fueron tres: La primera fueron los resultados de la investigación, en particular las carencias detectadas para el desarrollo del modelo de educación intercultural bilingüe. La segunda fueron las observaciones y las propuestas que los profesores bilingües participantes realizaron en el marco de entrevistas, grupos de discusión y talleres formativos. Entre estas destacaron las técnicas y recursos de enseñanza que cada uno empleaba en situaciones diversas. Se incorporaron a la guía para facilitar el aprendizaje de los alumnos. Esta medida les permitió sentirse autores de la mis-

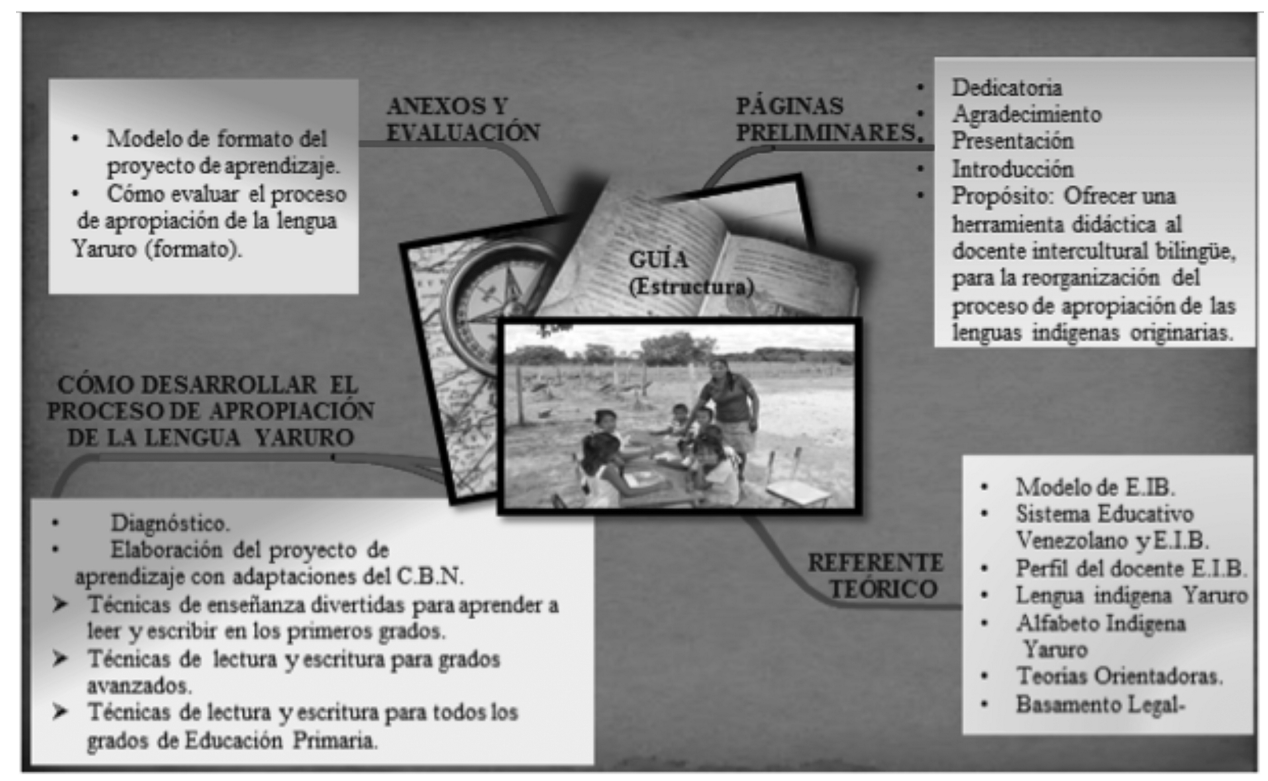

Gráfico $N^{o}$ 3: Estructura de la guía didáctica 
ma, lo que resultó clave para su aceptación, desarrollo y continuidad más allá del proceso de investigación, en sentido estricto. La tercera fuente fue el currículo oficial para la etapa y su adaptación al entorno intercultural bilingüe pumé.

Pretendía fundamentar la enseñanza y facilitar la apropiación del yaruro de un modo más eficaz. Fue previamente validada con expertos y ajustada en su diseño por su intervención. La versión final quedó estructurada en cuatro partes: $1^{\mathrm{a}}$ ) Páginas preliminares: presentación, introducción y propósito. $2^{\mathrm{a}}$ ) Referente teórico. $3^{\mathrm{a}}$ ) Cómo desarrollar el proceso de apropiación de la lengua yaruro, y $4^{\mathrm{a}}$ ) Anexos y evaluación. Los parágrafos más relevantes de su tercera parte hacían referencia al diagnóstico pedagógico de las debilidades y fortalezas en el dominio de la lengua originaria, y a la elaboración de proyectos de enseñanza-aprendizaje desde el Currículo Básico Nacional, incorporando tanto contenidos del área "Lenguaje, comunicación y cultura" (estructuras gramaticales) como de todas las áreas, así como saberes ancestrales. Además, enfatizaba en la aplicación de técnicas y recursos de enseñanza creativa y motivadora. Las técnicas didácticas se clasificaron en tres tipos: para los primeros grados, para grados avanzados y para grados mixtos.

La guía fue objeto de un taller formativo realizado con los profesores durante el tercer año y de otras sesiones desarrolladas durante el proceso de enseñanza. Tras su aplicación fue evaluada tomando como referencia sus objetivos y resultados, desde técnicas de evaluación cualitativa como la observación sistemática, la entrevista individual, el focus group (Krueger, 1988) y los grupos de discusión con alumnos y con padres y madres de alumnos.

Los alumnos se mostraron más participativos, disfrutaban con las técnicas de enseñanza y con los recursos de aprendizaje, considerándolos como juegos que les permitían compartir y construir conocimientos. Una vez triangulados, los resultados de la evaluación la confirmaron como un buen recurso para el cambio y la mejora de la enseñanza del yaruro, con posibilidades de servir en

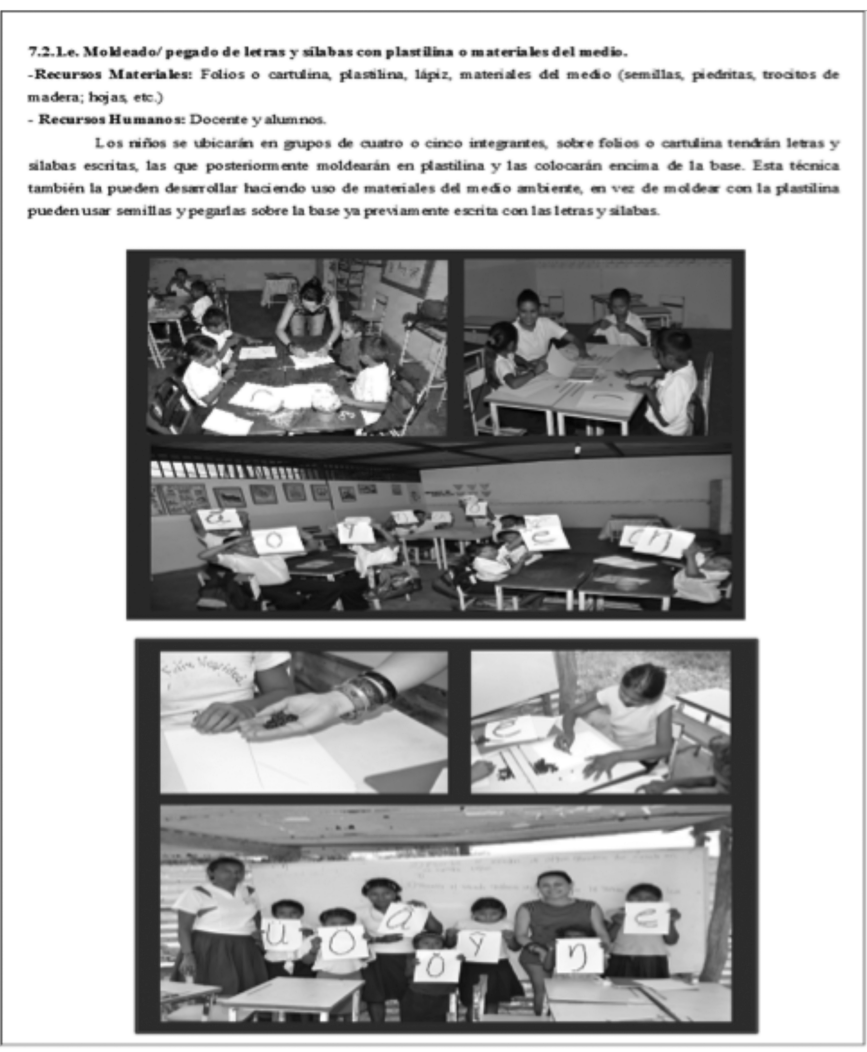

Gráfico $N^{o}$ 4. Ejemplo de la Guía Didáctica 
otros entornos indígenas latinoamericanos con lengua originaria propia. Al término del proceso investigador, los conocimientos de la guía continuaron siendo desarrollados en las escuelas pumé, consolidándose los logros ya observados: mejora del clima didáctico e incluso mayor colaboración entre docentes. Por ejemplo, se inició una práctica formativa en la que los maestros que dominaban la lengua indígena se integraron en las aulas de los que no lo hacían para intercambiar conocimientos y dialogar sobre el desarrollo de la comunicación didáctica bilingüe. A continuación se muestra un ejemplo (fragmento) de la guía didáctica.

\section{Discusión Y CONCLUSIONES}

El proceso de investigación permitió llegar a un conjunto interrelacionado de conclusiones.

Pese a que el currículo oficial de Venezuela considera que la educación intercultural bilingüe como "una modalidad que transversaliza [sic] el sistema educativo nacional", en las siete escuelas de la Red "Guaicaipuro" esta educación no existe. Para los pumé no hay nada parecido a "un referente ideológico, cultural y filosófico que guíe el proceso educativo de los pueblos indígenas" (Alonso, 2006: 56). Actualmente tampoco cuenta con las bases imprescindibles para su desarrollo futuro.

El docente bilingüe de estas escuelas actúa por sentido común y sin un modelo orientativo compartido. El Ministerio de Educación no desarrolla programas de actualización docente, ni le proporciona herramientas necesarias. Tampoco dispone de apoyos, recursos, libros de texto o guías docentes. El resultado es un docente desactualizado que realiza adecuaciones curriculares inadecuadas y pobres de un currículum alejado de la realidad indígena. Estas conclusiones coinciden con la investigación de Travé (2013), que evidenció que la falta de formación del profesorado en el conocimiento del idioma, en lingüística y en metodología didáctica son las limitaciones más significativas de los centros bilingües que inciden en el desarrollo de los alumnos. En nuestro estudio, la mayoría de los docentes de las escuelas indígenas tienen formación académica, pero no pedagógica, ni en educación intercultural bilingüe ni en el yaruro y su didáctica. Además, su perfil motivacional no está cimentado en el compromiso cultural con la causas del pueblo pumé, en la estimación de lo diferente ni en el dominio de la lengua materna de sus educandos. Aunque hay maestros que dominan el yaruro de forma oral, no poseen el dominio de las competencias lectora y escritora necesarias para su educación formal. Todo ello condiciona la práctica docente y limita la función de la escuela en el entorno indígena.

En el proceso de interpretación crítica del modelo de educación intercultural bilingüe realizada por los profesores bilingües, destacó una dificultad evaluativa. Concretamente, la relativa a percibir barreras y limitaciones radicadas en su propia formación. Pero, por otro lado, su participación permitió generar propuestas con las que se pudo elaborar, aplicar y evaluar la guía didáctica. Una vez elaborada e interiorizada fue plenamente aceptada por los profesionales. Su aplicación y evaluación nos permite concluir que cuanto más didáctico y fundado es el proceso de enseñanza intercultural bilingüe —en la identidad cultural, la lengua indígena, la integración escuela-comunidad, las técnicas y recursos didácticos, la praxis pedagógica, los contenidos de aprendizaje y la evaluación-, mejor es la apropiación de la lengua y más duraderos son los resultados formativos en los alumnos. 
La educación intercultural bilingüe depende además del vínculo comunidad-escuela. De la observación de los problemas didácticos, lingüísticos y culturales de la comunidad pumé y de su escuela se deduce la necesidad de colaboración de los implicados para responder globalmente a las necesidades sistémicas. Como punto para apoyar la palanca del cambio, los investigadores destacan el papel real y potencial de los ancianos como autoridades naturales aceptadas por la comunidad. Además, son quienes mejor hablan, escuchan, leen y escriben el yaruro. Su papel de expertos pudiera aprovecharse para intercambios formativos con los profesores bilingües.

Los aspectos mencionados en párrafos anteriores caracterizan la guía como un producto de interrelación directa con los protagonistas del entorno estudiado. Su particularidad y novedad consisten no sólo en su elaboración, sino en su aplicación y evaluación, cuyos resultados manifiestan su funcionalidad y pertinencia con la comunidad. Por otro lado, su contenido y redacción la permiten ser adaptada y utilizada como referente en el proceso de apropiación de lenguas originarias en elámbito indígena latinoamericano.

\section{Referencias Bibliográficas}

Abdallah-Pretceille, M. (1986). Vers une pédagogie interculturelle. Paris: Presses de la Sorbonne. Abdallah-Pretceille, M. (1996). Éducation et communication interculturelle. Paris: PUF.

Alonso, A. (2006). Modelo educativo del bachillerato intercultural. México: SEP.

Bartolomé, M. (2001). La construcción de la identidad en contextos multiculturales. Madrid: CIDE.

Bondarenko, N. (2010). Lenguas Minoritarias de Venezuela: Consideraciones desde la Perspectiva Ecolingüística, en Filolofía y Lingüistica, 36, 1: 175-189.

Bryant, A. y Charmaz, K. (eds.) (2007). The SAGE handbook of grounded theory. London: Sage Publications.

Busot, A. (1991). Investigación educativa. Maracaibo: EdiLuz.

Calvet, L. (1999). Pour una Écologie des Langues du Monde. Paris: Plon.

Camilleri, C. \& Cohen-Emerique, M. (eds.) (1989). Chocs de cultures: concepts et enjeux pratiques de l'interculturel. Paris: L'Harmattan.

Casal, A. (2006). Pasos para una investigación. Caracas: Santillana.

Castillo, S. (2008). La producción socioeducativa del conocimiento de las comunidades indígenas pumé. Tesis Doctoral no publicada, Universidad Nacional Experimental Simón Rodríguez. San Fernando, Venezuela.

Corbin, J. y Strauss, A. (1990). "Grounded Theory Research: Procedures, Canons, and Evaluative Criteria”, in Qualitative Sociology, 13, 1: 3-21.

Demorgon, J., Lipianski, E.-M. (1999). Guide de l'interculturel en formation. Paris: Retz.

Dey, I. (1999). Grounding grounded theory: guidelines for qualitative inquiry. San Diego, CA: Academic Press.

España, M. (2008). Modelo de educación multicultural indigena. Aportes teóricos desde una perspectiva de cointegración socio educativa. Tesis Doctoral no publicada, Universidad Nacional Experimental Simón Rodríguez. San Fernando, Venezuela.

García de C., F. (2004). El cuestionario: Recomendaciones metodológicas para el diseño de un cuestionario. Córdoba: Limusa.

García, J. (2009). Educación intercultural y profesorado. Caracas: Santillana.

Glaser, B. (1978). Theoretical Sensitivity: Advances in the Methodology of Grounded Theory. Mill Valley, CA: The Sociology Press. 
Gómez, D. (2010). "Cambio y mejora en las organizaciones educativas", en Educar, 47, 1: 31-39. Hammersley, M. y Atkinson, P. (1994). Etnografía. Métodos de investigación. Barcelona: Paidós. Haugen, E. (1972). "The ecology of language". in A. Dil (ed.), Essays by Einar Haugen. Stanford: Stanford University Press, 325-339.

Hernández, R., Fernández, C. y Baptista, P. (2006). Métodos y técnicas de la investigación II. México: McGraw-Hill.

Herrán, A. de la (2011). "Reflexiones para una reforma profunda de la educación desde un enfoque basado en la complejidad, la universalidad y la conciencia", en Educación XX1, 14.1: 245-264, available from: http://www.uned.es/educacionXX1/, accessed 19 August, 2013.

Herrán, A. de la y Paredes, J. (2009). La práctica de la innovación educativa. Madrid: Síntesis. Husserl, E. (1990). Invitación a la fenomenología. Barcelona: Paidós.

Jiménez, Y. (2005). El proceso cultural en educación escolarizada. Educación bilingüe intercultural en los zapotecos de Oaxaca (México). Granada: Universidad de Granada http:// bvirtual.proeibandes.org/bvirtual/docs/t_yjimenez.pdf, acceso 10 de noviembre de 2011.

Junta de Extremadura, Consejería de Educación y Cultura. (2011). Plan Marco de Atención a la Diversidad. Disponible en: http://sauce.pntic.mec.es/ falcon/planatd.pdf, acceso 10 de noviembre de 2012.

Krueger, R. (1988). Focus Groups: A practical guide for applied research. California: Sage.

Lamus, L. (2005). Estudio de interculturalidad y bilingüismo en el contexto de la praxis educativa en la comunidad indigena Piaroa. Tesis Doctoral no publicada. Universidad de Barcelona, Barcelona.

Leal, G. J. (2005). La autonomía del sujeto investigador y la metodología de investigación. Mérida: Litorama.

Leal, L. C. (2010). "Usos del concepto raza en Colombia", en R. Mosquera, A. Laó y C. Rodríguez (eds.), Debates sobre ciudadanía y politicas raciales en las Américas negras. Bogotá: Universidad Nacional de Colombia, 389-438.

Magro, M., Fernández, M. E. y Meza, M. (1996). Una visión multicultural para formar en la diversidad. Tesis Doctoral no publicada. Universidad Nacional Experimental Simón Rodríguez, Caracas, Venezuela.

Marshall, C. y Rossman, G. (1995). "Designing qualititive research", in A. O. Scribano (ed.), El proceso de investigación cualitativo. Buenos Aires: Prometeos, 33-35.

Martínez L. M. (2007). "El nuevo papel del profesor universitario de lenguas extranjeras en el proceso de convergencia europea y su relación con la interacción, la tutoría y el aprendizaje autónomo", en Porta Linguarum, 7:31-43, available from: http://www.ugr.es/local/ portalin, accessed 19 August, 2013.

Martínez, M. (1991). La inercia mental en los estudios de postgrado. Caracas: Argos

Martínez, M. (2004). Ciencia y arte de la metodología cualitativa. Guadalajara: Trillas.

Mato, T. (2006). "Interculturalidad, producción de conocimiento y prácticas educativas". Caracas: Consejo Latinoamericano de Ciencias Sociales CLACSO , 120-138. Universidad Central de Venezuela.

Mena, A. M. (2009). Imaginando un cambio en las prácticas educativas de los profesores de la Facultad de Ciencias. Tesis de maestría no publicada, Universidad Autónoma de San Luis Potosí, San Luis de Potosí (México).

Mirabal, D. y Laya, C. (2004). Influencia de la cultura Yaruro y la cultura apureña en la historia de la vida de Chela Migdalia Páez. Tesis Doctoral no publicada. Universidad Nacional Experimental Simón Rodríguez, San Fernando, Venezuela.

Montecinos, C. (2004). Educación multicultural. Nuevos sentidos para la Pedagogía (2a ed.). Talca: Universidad de Talca y Departamento de Educación. Universidad de la Frontera. 
Patton, M. (1990). Evaluación cualitativa y métodos de investigación. Beverly Hills: Sage.

Ramos, G. A. M. (2010). "El reto de enseñar una lengua extranjera sin recursos", en Revista Porta Linguarum 14: 79-89, available from: http://www.ugr.es/local/portalin, accessed 19 August, 2013.

Ruiz, D. (1996). La alfabetización temprana en el ambiente preescolar. San Juan: Universidad de Puerto Rico.

Sánchez, C. (2006). Educación en valores interculturales. Tesis Doctoral no publicada, Universidad de Granada.

Stuart, H. (1996). Questions of Cultural Identity. London: Sage.

Taylor, S. y Bogdan, R. (2000). Introducción a los métodos de investigación cualitativa. La búsqueda de significados. Barcelona: Paidós.

Travé G. (2013). "Un estudio sobre las representaciones del profesorado de Educación Primaria acerca de la enseñanza bilingüe", en Revista de Educación, 361: 379-402, available from: http://www.mecd.gob.es/revista-de-educacion, accessed 19 August, 2013.

Villasante, T.R. (2006). Desbordes creativos. Estilos y estrategias para la transformación social. Madrid: La Catarata.

Williamson, G. (2004). "Educación Multicultural, Educación Multicultural Bilingüe, Educación Indígena o Educación Intercultural", en Cuadernos Interculturales, 2: 3, 25-33.

Wilson L., F. (2005). Técnicas de aprendizaje. Cali: Universidad de Cali. 